\title{
La Responsabilidad Social Universitaria: emprendimiento sostenible como impacto de intervención en comunidades vulnerables
}

FECHA DE RECEPCIÓN: 27 de julio

FECHA DE APROBACIÓN: 26 de agosto

Pp.

\section{Resumen}

El propósito del estudio es diseñar un programa de responsabilidad social universitaria en CORPOSUCRE para generar emprendimiento sostenible en comunidades vulnerables; en la construcción de los referentes se analizaron los enfoques de Vallaeys (2010), Bernal \& Rivera (2011) y Rodríguez (2012). La investigación fue orientada desde un paradigma de investigación mixto; ésta, se desarrolló mediante el estudio e intervención que ha hecho la Corporación Universitaria Antonio José de Sucre con varios grupos de interés, así como también con la recolección de información mediante un cuestionario estructurado que se aplicó a los líderes de la comunidad Altos de la Sabana, por lo que se identifica como resultado preliminar, que en CORPOSUCRE existe conciencia acerca de la importancia de la proyección social, sin embargo, los funcionarios de la corporación aún perciben la responsabilidad social universitaria, como un proceso aislado y discrecional, por lo tanto se promueve el diseño de un programa de RSU.

\section{Palabras clave}

Responsabilidad, universidad, emprendimiento sostenible, vulnerabilidad social, comunidad.

\footnotetext{
Yamaru Valle Chirinos Araque

Doctora en Ciencias Mencion Gerencia de la Universidad Privada Doctor Rafael Belloso Chacin; magíster en Docencia para la Educación Superior en la Universidad Nacional Experimental Rafael Maria Baralt. Docente investigadora titular de la Corporación Universitaria Antonio José de Sucre CORPOSUCRE.
}

\section{Claudia Milena Pérez Peralta}

Magíster en Educación, Universidad de Sucre. Docente titular, Corporación Universitaria Antonio José de Sucre - Corposucre 


\title{
Educational leadership in the 21st century from the perspective of sustainable entrepreneurship
}

\begin{abstract}
Abstrac. The purpose of the study is to design a program of university social responsibility in CORPOSUCRE to generate sustainable entrepreneurship in vulnerable communities; in building referents approaches Vallaeys (2010) analyzed, Bernal \& Rivera (2011) and Rodriguez (2012). The research was oriented from a paradigm of joint research; it was developed by studying and intervention that has made the University Corporation Antonio Jose de Sucre with various interest groups, as well as the collection of information through a structured questionnaire that leaders Altos community of Sabana applied, which is identified as preliminary result, there is awareness CORPOSUCRE about the importance of social projection, however, officials of the corporation still perceive the university social responsibility as an isolated and discretionary process, therefore it promotes the design of a program of RSU.
\end{abstract}

Key words. Responsibility, university, sustainable entrepreneurship, social vulnerability, community.

\section{La responsabilité sociale universitaire : l'entrepreneuriat équitable et son impact sur les populations vulnérables}

Resumé. L'objectif de cette étude réside dans la création d'un programme de responsabilité sociale universitaire (RSU) offert par I'université CORPOSUCRE qui a pour but de générer des programmes d'entrepreneuriat équitable destinés à des communautés vulnérables. Cette étude se base sur l'analyse de la pensée des auteurs Vallaeys (2010), Bernal \& Rivera (2011) et Rodríguez (2012). L'investigation est orientée vers un paradigme de type mixte réalisé grâce aux interventions de la Corporation Universitaire Antonio José de Sucre envers certaines de ces populations. Le recueil d'informations et résultats préliminaires de l'étude ont été réalisés au moyen d'un questionnaire structuré et présenté aux représentants de la communauté Altos de la Sabana. Notons enfin que l'université CORPOSUCRE a conscience de l'importance de la protection sociale des populations vulnérables alors que la notion de responsabilité sociale universitaire (RSU) est encore perçue par les employés de l'institution comme un processus isolé et discrétionnaire nécessitant la création d'un programme spécifique de responsabilité sociale universitaire (RSU).

Mots clefs. Responsabilité, université, entrepreneuriat équitable, vulnérabilité sociale, communauté. 


\section{Introducción}

In las sociedades modernas, la universidad 1 debe trascender desde su medio interno y autónomo, hacia una posición abierta a influencias externas, desde una relación recíproca, de constante retroalimentación con el medio circundante. En este entorno, la responsabilidad de las instituciones educación superior (IES) adquiere dos connotaciones; la primera se refiere al logro de la eficiencia en el cumplimiento de las funciones, lo que se denomina gestión de la calidad universitaria, y la segunda, hace énfasis en el cumplimiento de las funciones en el marco de los requerimientos y de la dinámica de la sociedad, lo que se define como pertinencia (Bernal \& Rivera, 2011). Según Rodríguez (2012), la responsabilidad social universitaria (RSU) es una realidad inherente a la naturaleza y misión del nivel de educación superior.
En efecto Vallaeys (2007), considera que la universidad tiene una responsabilidad con la sociedad, no solo desdesus objetivosmisionales, es decir, con la formación académica que brinda, la generación de nuevo conocimiento, la proyección social o extensión; sino también, a nivel interno, desde su incidencia dentro de su misma organización, como generadora de empleo y calidad de vida para sus trabajadores; en a este respeto, la IES impactan, no solo desde la generación de capital humano, sino, desde la gestación de capital social (Vallaeys, 2010). La responsabilidad social de las universidades más que generar impactos educativos, cognitivos, de innovación o de investigación, genera impactos sociales así como, organizacionales, que se gestan desde varios actores e inciden en distintos grupos de interés. En tal sentido, se distingue un diagrama del autor antes mencionado donde se observa la interacción entre impactos y actores sociales:

Figura 1. Impactos, Actores y Grupos de Interés de la Responsabilidad Social Universitaria - RSU

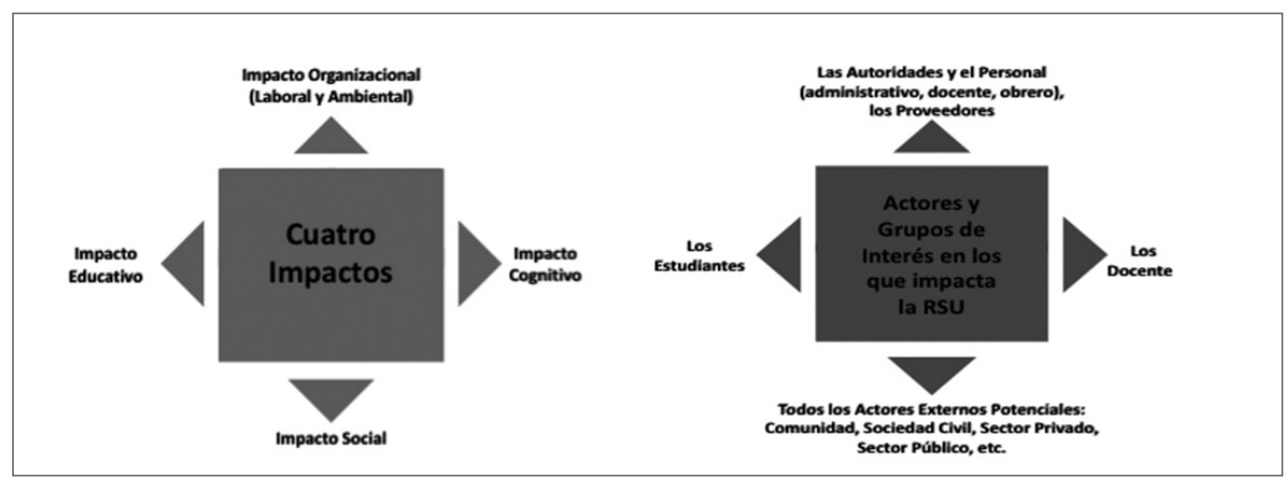

Fuente. Vallaeys, 2010.

Continuando con las ideas del autor antes mencionado se explica que las universidades desde sus procesos académicos, la universidad reconoce varias dimensiones que configuran aspectos fundamentales en la construcción de conocimiento (Naidorf, Giordana \& Horn, 2007), siendo la educación su principal objetivo misional. Las economías en el mundo, basan su desarrollo en la mayor disponibilidad de conocimientos e innovaciones tecnológicas, donde, la información se han instaurado como una nueva fuerza productiva, la cual, hace parte del capital humano y social (Tünnermann \& De Souza, 2003); esta perspectiva se ha 
configurado en un espacio llamado hoy en día sociedad del conocimiento, que establece nuevas relaciones entre el saber teórico, la sociedad y la política (Marrero, 2007), y exige de las universidades mayor compromiso no solo en la generación de saberes, y capacidades que susciten el cambio social.

Por lo tanto la universidad desde su trayectoria histórica, y las nuevas realidades, adquiere un compromiso para aportar en la transformación social, apoyando al colectivo, de manera responsable, al suministrarle herramientas, e incentivos para la generación de nuevas formas, que le representen realidades más favorables; sobre todo, si se trata de grupos poblacionales en situaciones de desventaja económica y exclusión social. El emprendimiento sostenible, constituye una forma de lograr inclusión laboral en comunidades que han sido mal catalogadas comovulnerables, considerándoseéstecomoun fenómeno deseable, que empodera y dignifica la supervivencia del ser humano; el mismo se construye desde programas de intervención social que favorezcan o potencialicen este tipo de actividades económicas, lo cual, es fundamental para impulsar la economía y el bienestar social (Chirinos, 2014).

Asi que teniendo en cuenta las condiciones adversas en el entorno para acceder a un empleo de calidad, sobre todo para la población vulnerable, una buena estrategia que permite subsanar esta situación, es la generación de emprendimientos sostenibles, donde, no solo se generaría autoempleo, sino también trabajo para otras personas. Según Sparano (2014), el emprendimiento es un proceso que con el transcurso del tiempo causa un impacto positivo en la creación de empresas, lo que posibilita la generación de empleos en los distintos países. En Colombia, según el informe Global Entrepreneurship Monitor - GEM para
2014, la Tasa de Actividad Emprendedora - TEA fue de 18,5\%, una de las tasas más bajas de América Latina; según este estudio, a pesar que en el país existe actitud de parte de la población para la generación de nuevas emprendimientos (Uninorte, 2016), hay muchas barreras de contexto e institucionales que limitan su acción, éstas están principalmente relacionadas con: las exigencias burocráticas, los aspectos legales, las obligaciones tributarias y las necesidades financieras así como de inversión (Correa, 2011); lo que sumado a que la política de apoyo que solo se centra en el nacimiento de las nuevas empresas y no en su mantenimiento (Kantis, Federido \& Menéndez, 2012), hacen que este tipo de negocios sean de corta duración.

Continuando con el enfoque anterior, se deben adelantar programas de parte de las universidadescomoforma desu responsabilidad social, que podrían clasificar e identificar a los emprendedores potenciales, con el objetivo de optimizar las probabilidades de éxito en sus iniciativas de negocio garantizando estabilidad económica a la sociedad, mejorando su calidad de vida, conduciendo al desarrollo integral. Sin duda la optimización de las posibilidades para los emprendedores estimula los programas de desarrollo local y es un caldo de cultivo para el empleo, esto es aún más acuciante cuando se ha destacado ciertas habilidades directivas en las personas emprendedoras parece tener un efecto drástico en el crecimiento y permanencia de las de nueva creación (Fuller, 2006).

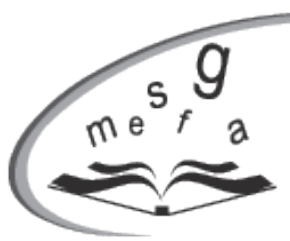

Es relevante mencionar la necesidad de promover el espíritu emprendedor y de responsabilidad social en todas las instituciones educación superior, por tal razón, debe ser apoyado en un marco interinstitucional que 


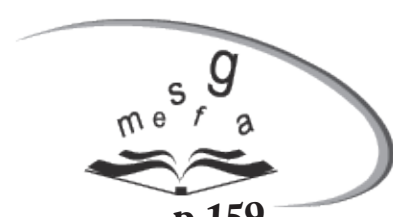

permita fomentar, así como, desarrollar la cultura del emprendimiento sostenible, estableciendo un vínculo entre el sistema educativo y sistema productivo, mediante la formación de competencias empresariales a través de una cátedra transversal de emprendimiento, así como, generar redes regionales que fortalezcan el proceso de manera local (Chirinos, 2014).

La presente investigación, corresponde desde sí misma, a la conjugación de los cuatro impactos que configuran la responsabilidad social universitaria; es investigación, desde la indagación de estados del arte, así como, el diagnóstico de contextos particulares; de otro lado, el trabajo genera procesos de proyección e impacto social, así como educativo, desde la incidencia en la formación de nuevos emprendedores, líderes y ciudadanos, lo cual, es una acción que acerca la docencia hacia la comunidad externa. Constituye además un impacto organizacional en la medida que generará nuevas formas en la comunidad universitaria de la Corporación, para desarrollar su responsabilidad social, mediante el programa estratégico que se va a formular, constituyendo esto, un compendio de saberes contextualizados en realidades complejas.

Por consiguiente la razón de ser de toda esta dinámica está fundamentada en el compromiso que tienen las universidades con el desarrollo social, en particular con comunidades vulnerables, se debe comprometer a contribuir en la generación de procesos de inclusión social. Para que ésta se genere, se deben dar oportunidades para todas y todos (Cordero, 2011), y una forma de generarlas a nivel laboral son los emprendimientos sostenibles (Ishikawa \& Strandberg, 2009); las universidades pueden propiciar espacios para la conformación de este tipo de negocios, a partir, de la concepción de emprendimientos sociales, que mediante procesos de innovación social, pueden generar desarrollo sostenible en las comunidades (Sánchez, 2008). Es importante resaltar que la sinergia entre el Estado, instituciones educativas, las empresas públicas y privada, para que apoyen las iniciativas de negocios en comunidades vulnerables debido que estas comunidades necesitan mucha asistencia, asesorías y guas para poder generar emprendimientos sostenibles de tal modo mejorar la calidad de vida de todo la sociedad.

De acuerdo con lo planteado se justifica esta investigación cuyo objetivo es diseñar un programa de responsabilidad social universitaria en CORPOSUCRE para generar emprendimiento sostenible en comunidades vulnerables, de esta forma se está coadyuvando a que las mismas surjan prósperamente $y$ tengan una mejor calidad de vida, ya que mediante la incorporación de la universidad socialmente responsable tendrán herramientas para crear nuevas iniciativas de negocios que los lleve hacia la inclusión laboral dentro de su propia comunidad. 


\section{Referentes teóricos}

\subsection{La responsabilidad social universitaria: un concepto amplio}

Según Misas (2004), se hace necesario develar la conexión entre el conocimiento y la producción, es en este escenario, donde la investigación la generación de nuevos avances técnicos y tecnológicos, pueden traducirse en nuevos saberes así como formas, esto, a su vez en riqueza social. Las IES se proyectan más allá de su entorno cercano hacia el contexto. A menudo, se ha venido consolidando la discusión en torno a la extensión universitaria; con respecto a esto, en algunos países se han incorporado estos temas en sus sistemas de evaluación, certificación así como acreditación (Bernal \& Rivera, 2011). La extensión universitaria conjuga elementos tan importantes como: el trabajo de participación, promoción humana comunitaria, las alianzas estratégicas entre la sociedad civil, el estado, el sector privado, la academia, y el trabajo multi, inter y transdisciplinario desde distintas líneas de acción, en un proceso dialógico de retroalimentación constante.

Igualmente, la educación, se ha considerado como el pilar fundamental del desarrollo a nivel global, sin embargo el acceso a ella, aún sigue siendo, más que un derecho un privilegio (UNESCO, 2008); en algunos países inclusión a la universidad tiene dificultades, donde, su patrón de crecimiento no es equitativo (Rama, 2007). Así mismo ocurre con la investigación, ya que la inversión es mínima desde el sector público, por consiguiente, cuando es hecha por instituciones privadas, los resultados no se democratizan, sino que permanece en la alta sociedad, perpetuándose así, estructuras de poder autoritarios (Suárez, 2007). De este modo se evidencia que la proyección universitaria, aún sigue siendo una actividad comúnmente separada de la realidad a nivel interuniversitario, e intrauniversitario, reduciéndose en diversas oportunidades a actividades asistencialistas (Bernal \& Rivera, 2011).

Afirma Valleys (2009), la responsabilidad social de las organizaciones es el despliegue de los valores éticos donde la organización se compromete a minimizar sus impactos negativos y a maximizar sus impactos positivos, trabajando para dar un servicio cada vez mejor para todos, reconociendo o diagnosticando cuáles son los impactos que en concreto (tanto internos como externos a la organización) crea la universidad en su entorno, en función de responsabilizarse de los mismos para que sean o resulten positivos en vez de negativos y gestionarlos, de manera que, recomienda gestionar los impactos que generan las IES en el despliegue de la responsabilidad social, en vez de realizar una política de promoción de valores.

Así mismo, el autor antes mencionado diseña una tipología de los impactos que deben ser gestionados por las instituciones de educación superior (IES), las cuales deben estar conscientes de los mensajes ocultos dirigidos a los estudiantes y a la sociedad se clasifica de la siguiente manera:

\section{- Impacto organizacional: laboral y ambiental}

Constituyen los impactos generados en su personal, producto de las políticas de bienestar social y la contaminación del medio ambiente (desechos, deforestación, polución atmosférica por transporte vehicular, etc.). 


\section{- Impacto educativo}

Hace referencia a la formación de los jóvenes y profesionales, a su manera de interpretar e imaginar el mundo, comportarse en él y valorar ciertas cosas más que otras. Influye asimismo sobre la deontología profesional, orienta (de modo consciente o no) la definición de la ética profesional de cada disciplina y su rol social. Esta responsabilidad, puede llevar a la universidad a promover, desde su misma malla curricular, una educación desligada de los problemas sociales, con fines solamente instrumentales (con interés de obtener sólo el grado universitario), sobre la base de una relación pedagógica asimétrica profesor/alumno, sin afán de formación integral ciudadana ni promoción de la alegría de aprender.

\section{- Impacto cognitivo}

El impacto cognitivo es de carácter científico y epistemológico, dado que la Universidad orienta la producción del saber y las tecnologías, e influye en la definición de lo que se llama socialmente verdad, ciencia, objetividad, racionalidad, legitimidad, utilidad, igualmente, participa en la delimitación de los ámbitos de cada especialidad, así como en la definición de los problemas en la agenda de las ciencias, al igual, que articula la relación entre tecnociencia y sociedad y maneja en gran medida la gestión del conocimiento.

\section{- Impacto social}

Además de los mensajes ocultos que la universidad envía a sus comunidades internas y externas, tiene un impacto sobre la sociedad y su desarrollo económico, social y político, no sólo forma a los futuros líderes y profesionales de la nación, sino que es también un referente, una fuente de legitimación, y un actor social.
El autor antes mencionado, indica que la responsabilidad social fundamentada en la gestión de Impactos, ofrece un marco de actuación más favorable que la promoción de valores: Porque obliga a preguntarse: ¿quiénes somos y de qué malos impactos en el entorno somos responsables? (obliga a analizar nuestro actuar oculto), porque obliga a ver la responsabilidad social en términos de estrategia gerencial y no como actividad marginal de la universidad, porque invita a practicar una topofilia compleja así como holística y no sólo una filantropía bidireccional de tal modo, se concibe en términos de Desarrollo y nunca como asistencialismo (que deseduca a los estudiantes), porque transforma la virtud de solidaridad en aprendizaje permanente de las intersolidaridades del mundo, haciendo cada vez más inteligente la ética.

\subsection{El concepto de emprendimiento sostenible}

El emprendimiento sostenible se caracteriza por ser una forma de integración social, humana y económica, con la finalidad de realizar iniciativas de negocios que permitan el aprovechamiento de las oportunidades que se presentan en el entorno, asímismo se identifica por buscar la mejor forma de producir bienes y servicios que satisfagan las necesidades de la colectividad, incorporando a su actividad la creatividad así como la innovación, otra de sus características relevantes es tomar al individuo como

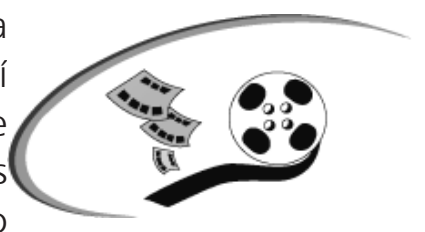
valor agregado de competencia, para el desarrollo integral del entorno económico, logrando así una estabilidad que garantice su sostenibilidad, esta nueva visión de emprendimiento sostenible por su carácter innovador, viene a desplazar la concepción tradicional donde el beneficio es aprovechado 
de forma individualista, de empoderamiento económico capitalista, sin pensar en el hombre como ser inteligente capaz de crear e innovar y trasformar su realidad, desde esta perspectiva el mismo es visto como simple mano de obra para explotar creando fortuna para unos pocos (Chirinos, 2014)

Por tanto, el emprendimiento sostenible vendrá a conglomerar un conjunto heterogéneo de iniciativas destinadas a generar de forma paralela bienes económicos, sociales y ecológicos (López, 2012). En otras palabras, desde el punto de vista de la investigación social el emprendimiento sostenible es asumido e identificado como el proceso de estudio de quien y como son descubiertas creadas, ordenadas así como explotadas las oportunidades, para traer a la existencia futuros bienes y servicios teniendo en cuenta sus consecuencias económicas, sociales también medio ambientales (Cohen y Franco, 2005).

\subsection{La universidad generadora de emprendimiento sostenible}

Es relevante destacar la influencia de las universidades como entes generadoras de conocimiento donde se estudie la mejor opción, para implementar planes o programas que incentiven a los individuos a desarrollar por voluntad propia, de forma responsable y con plenos conocimientos tanto científicos, técnicos y empíricos emprendimientos sostenibles. Por lo tanto el Estado es responsable de propiciar el ambiente para desarrollar el emprendimiento sostenible ya que este se articula como mecanismo al sistema político para la generación de desarrollo económico y social en el país (Chirinos, 2014).

De tal manera que incorporar las universidades es de suma importancia para facilitar el proceso de crear cultura de innovación, incrementar el pensamiento creativo y trasformador en los individuos, por tanto es necesario incluir cambios en el sistema de educativo en todos sus niveles, con la finalidad de impulsar el desarrollo de emprendimiento sostenible, que sea visto como una opción de crecimiento económico (Chirinos \& Ramos, 2014).

Así mismo, promover redes entre empresas públicas y privadas para fortalecer las iniciativas nacientes, que interactúen entre sí generando conocimientos innovadores, compartiendo experiencias, de este modo tener una visión amplia para el mejor aprovechamiento de oportunidades con la finalidad de convertirlas en procesos productivos o en bienes y servicios para beneficiar al colectivo (Chirinos, 2014).

Por lo tanto es importante implementar mecanismos de participación como una necesidad y oportunidad para que el Estado pueda involucrar a la sociedad civil y las iniciativas de negocios nacientes, en acciones de cooperación de tal manera que puedan avanzar en la búsqueda de generación de nuevos empleos, aportando significativamente al mejoramiento de la calidad de vida de los individuos, apuntando a una economía solidaria, que permita aprovechar el potencial existente para lograr transformación en la sociedad (Chirinos, 2014).

Según los postulados de Vicens y Grullón, (2011), la naturaleza social del proceso de innovación es más que fundamentarse en el tratamiento de los asuntos tecnológicos materiales, está fuertemente asociada con las habilidades individuales y colectivas para aprender a estar alerta ante las oportunidades, desarrollar competencias para poner en práctica las ideas de tal modo que las universidades deben vincular espacios generadores de conocimientos donde se 
adquieran esas cualidades y capacidades para lograr emprendimientos sostenibles que lleven a la contribución del desarrollo económico.

En tal sentido es importante destacar que es esencial el aporte y participación activa de las universidades como pilar fundamental en la formación del hombre nuevo como primordial agente en el desarrollo de este proceso, ya que genera conocimientos técnicos, científicos, tecnológicos, tan necesarios para el impulso de la innovación, por tal motivo se requiere de su apoyo en la implementación de planes así como programas dirigidos a la formación de emprendedores socialmente responsables, con principios y valores (Chirinos, 2014).

\subsection{Estrategias de responsabilidad social universitaria: generación de emprendimiento sostenible}

Es importante destacar que para lograr la transformación social e incrementar cambios en la visión de iniciativas de negocios y hacer que la misma tenga sentido de equidad e inclusión, la educación es pilar fundamental para la formación de emprendedores, la misma debe alimentar la vocación empresarial, propiciar la innovación, la creatividad, creando competencias individuales así como colectivas para desarrollar la cultura de innovación, poner de manifiesto los valores éticos de la sociedad, elementos estos tan necesarios para lograr el éxito del emprendimiento y su sostenibilidad.

Más aun cuando se evidencia la necesidad de las comunidades vulnerables en cuanto al apoyo por parte del de las universidades, de los

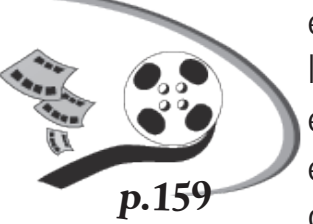
entes gubernamentales y de las empresas para realizar los emprendimiento sostenibles en sus diferentes etapas de desarrollo como es la fase de pre-incubación, incubación, financiamiento y consolidación, para lograr de esta forma la inclusión laboral, para evitar refugiarse en la informalidad.

Es por ello necesario replantear el modelo económico y financiero, educativo que estos incluyan programas responsables dirigidos a lo menos privilegiaos como son las comunidades vulnerables, de tal manera poder responder a los requerimientos exigidos por las iniciativas de negocio nacientes ya que el actual no está acorde con el dinamismo emprendedor sostenible, así trascender de un modelo insostenible a otro más idóneo para propiciar tecnologías sostenibles, modelos de negocios más justos y sus resultantes cambios en los estilos de vida de las personas.

En tal sentido la visión del emprendimiento sostenible en el escenario de lo posible debe incorporarse en el marco de la responsabilidad social universitaria ya que a través de este medio se pueden crear estrategias para lograr el desarrollo del mismo y garantizar su éxito, y está destinado a proporcionar valor a los seres humanos como principal protagonista del proceso emprendedor, incentivando a la mejor utilización de recursos tecnológicos avanzados y de producción propia, para que se adapte a los requerimientos exigidos por cada entorno, resaltando la importante de tomar como base la innovación que es el factor primordial para el desarrollo de las capacidades, habilidades y destrezas con las que se cuentan para lograr cambios y mejoras en la actividad del emprendimiento sostenible.

Paratal efecto, se requiere unfuertecompromiso por parte del Estado para transformar la sociedad, apoyando al colectivo, de manera responsable suministrándole herramientas con el fin de generar emprendimiento sostenible, logrando que este sea enmarcado en el modelo 
de desarrollo económico. Considerando que el emprendimiento sostenible es un fenómeno deseable, implementar políticas o programas de intervención social que favorezcan o potencialicen este tipo de actividad económica, es fundamental porque tiene como finalidad impulsar la economía y bienestar social.

Es relevante mencionar la necesidad de promover el espíritu emprendedor y de responsabilidad social en todas las instituciones educativas del país, por tal razón debe ser apoyado en un marco interinstitucional que permita fomentar así como desarrollar la cultura del emprendimiento sostenible, estableciendo un vínculo entre el sistema educativo y sistema

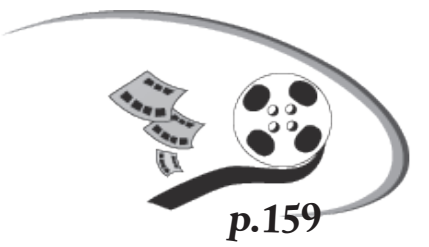

productivo nacional, mediante la formación de competencias empresariales a través de una cátedra transversal de emprendimiento así como generar redes regionales que fortalezcan el proceso de manera local.

Continuando con los planteamientos anteriores se identifica que es fundamental la formación integral en valores como desarrollo del ser humano y su comunidad, propiciando autoestima, autonomía, sentido de pertenencia a la comunidad, trabajo en equipo, solidaridad, desarrollo del gusto por la innovación, estímulo a la investigación, aprendizaje permanente, el reconocimiento de la conciencia, el derecho y la responsabilidad del desarrollo de las personas como individuos, como integrantes de una comunidad, apoyo a procesos de emprendimiento sostenibles desde la perspectiva social, cultural, ambiental y regional.

De esta manera, la actividad de crear nuevas empresas genera un importante valor añadido desde un punto de vista económico y social. Teniendo en cuenta que las iniciativas de negocio conllevan un efecto multiplicador así como de demostración, impulsando una cultura en la que conceptos como el ser, saber hacer, la innovación y la apuesta por el futuro cobran el protagonismo que merecen en la sociedad moderna.

Cabe destacar que para aumentar indiferenciadamente, la deseabilidad de iniciativas de negocios sostenibles hay que mejorar los mecanismos de la propiedad industrial e intelectual de las tecnologías sostenibles, simplificar los sistemas regulatorios y fiscales aplicar la ley asegurando transparencia, así como una buena gobernanza, reformando los servicios financieros, potenciando el rol de las universidades como nicho de fortalecimiento del emprendimiento sostenible y afianzando la relación entre las escuelas de emprendimiento e inversores. (Wheeler, y otros, 2005).

\subsection{Comunidades vulnerables: grupos de interés de la responsabilidad social universitaria}

La pobreza es un fenómeno complejo y multidimensional que implica carencia y falta de acceso al uso y aprovechamiento de recursos (Bueno, 2013); el riesgo que se presente este tipo de carencias, está relacionada con la presencia y severidad del nivel de vulnerabilidad social. Esta se refiere a la fragilidad o indefensión de un grupo de personas ante cambios del entorno; esta tiene lugar desamparo institucional, la debilidad interna del individuo para el aprovechamiento de oportunidades (Busso, 2001). La vulnerabilidad social está determinada por distintos aspectos de la vida de las personas tanto biológicos como condiciones de contexto (Espinosa, Calzoncit, Valdez \& Castro, 2012). 
En contraposición a la pobreza y a la vulnerabilidad social, està las políticas y estrategias para lograr calidad de vida en los grupos poblacionales. Según Blanco \& Chacón (1985), la calidad de vida, implica aspectos que trascienden de lo físicos, incluyendo áreas de bienestar social, relaciones sociales y acceso a los bienes culturales, y de más bienes que le proporcionen al individuo bienestar. Ferrans (1990b, citado en Urzúa \& CaqueoUrízar, 2012) define la calidad de vida como el bienestar personal derivado de la satisfacción o insatisfacción con áreas que son importantes para un individuo; mientras que Hornquist (1982, citado en Urzúa \& Caqueo-Urízar, 2012), la define como la satisfacción de las necesidades en las esferas física, psicológica, social, de actividades, material y estructural.

Por lo que para los gobiernos la mejora en la calidad de vida de los grupos poblacionales vulnerables, constituye un objetivo primordial, lo que implica la disposición de recursos de todo tipo y el abordaje de este tema desde una visión amplia, sobrepasando aspectos culturales y contextuales particulares. Para el caso de las empresas, incluyendo las universidades, el mejoramiento de las condiciones de vida de las comunidades implica mayor compromiso con este tipo de grupos sociales, y en particular generar en ellos capacidad, inteligencia colectiva, redes de apoyo y nuevas formas de afrontar sus propias realidades, donde el empoderamiento sostenible constituye una buena estrategia de desarrollo.

De tal manera que la preocupación por la situación de las comunidades vulnerables no debe estar focalizada únicamente en los gobiernos, si no en las organizaciones de todo tipo donde la responsabilidad social de estas como dimensión y valor ético inmanente a su razón de ser, debe constituir un aspecto central más que una actitud de tipo filantrópico, devolviendo de esta forma a la sociedad parte de sus propios beneficios. Esto implica un cambio de mentalidad, en el caso de las universidades el aporte que deben hacer desde sus posibilidades es mayor porque su orientación debe responder a satisfacer las necesidades del medio en el cual se encuentran inmersos. Las organizaciones deben generar desde su responsabilidad social valores sociales y humanos compartidos (García \& Rodríguez, 2011).

\section{Metodología}

$\checkmark$ ste estudio es de naturaleza cuanti1 cualitativa porque consiste, fundamentalmente en describir, estudiar y analizar un ámbito problemático específico, donde se identifican características propias del fenómeno. Hernández y Mendoza (2008) indican que los métodos mixtos representan un conjunto de procesos sistemáticos, empíricos y críticos de investigación e implican la recolección y el análisis de datos cuantitativos y cualitativos, así como su integración y discusión conjunta, para realizar inferencias producto de toda la información recabada (metainferencias) y lograr un mayor entendimiento del fenómeno bajo estudio. Según (Ander-Egg, 2008) define que el enfoque cuanti-cualitativo es aquello que permite reseñar las características o rasgos de la situación o fenómeno objeto de estudio.

En tal sentido la responsabilidad social universitaria: emprendimiento sostenible como impacto de intervención en comunidades vulnerables, cuyo objetivo fundamental es diseñar un programa de responsabilidad social universitaria en CORPOSUCRE para generar emprendimiento sostenible en comunidades 
vulnerables, se desarrolla bajo el enfoque antes mencionado debido a la complejidad del mimo y el carácter innovador que lo identifica, permite la fácil adaptación a este tipo de metodología la cual le imprime dinamismo, profundidad así como complejidad en la investigación lo que lleva a obtener resultados favorables que propicien el ambiente adecuado para ser aplicado, El tipo de investigación fue descriptivo, transeccional de campo.Según Hernández, Fernández y Baptista (2008) significa indagar la incidencia y los valores en que se manifiesta una o más variables. El procedimiento consiste en medir en un grupo de personas u objetos una o generalmente más variables y proporcionar su descripción. Se trata de la investigación aplicada para comprender y resolver alguna situación, necesidad o problema en un contexto determinado.

Por lo cual se aplicó un cuestionario diseñado por las investigadoras el cual fue aplicado a 54 líderes, de 68 que hay en toda la comunidad; recabándose información de 234 personas. El instrumento se estructuro de la siguiente manera, contiene dos (2) módulos, esenciales: la caracterización del hogar y las condiciones laborales. El cuestionario tuvo once (11) preguntas, que dieron respuesta a seis (6) variables fundamentales, específicamente aspectos sociodemográficos de los integrantes del hogar, así como, los aspectos de trabajo, formación, experiencia, habilidades y proyecto de vida laboral de las personas que trabajan en estos hogares caracterizados de esta forma se vincula la investigación cuantitativa. Es importante resaltar que también se aplicó para esta investigación la técnica de entrevista de informantes claves ya que se entrevistaron los representantes de proyección social de CORPOSUCRE y se hizo una revisión documental de todos los datos que tienen en actas y documentos propios dela creación de la proyección social, construcción y validación de datos, en esta parte se empleó el paquete estadístico SPSS, para procesar la base de datos, se hicieron pruebas de validación para encontrar fallas en la etapa de procesamiento; análisis de resultados y construcción de documentos.

\section{Resultados}

$\mathrm{E}$ n este apartado se exponen las características de los procesos de extensión social que ha llevado a cabo la Corporación Universitaria Antonio José de Sucre -Corposucre, así como los avances en materia de responsabilidad social universitaria para la generación de emprendimiento sostenibles en la comunidad Altos de la Sabana con la finalidad de diseñar un programa de responsabilidad social universitaria en CORPOSUCRE para generar emprendimiento sostenible en comunidades vulnerables. De tal manera la corporación concibe la proyección social, como el ejercicio a través del cual el quehacer académico se proyecta dinámica y coordinadamente, poniéndolo al servicio de la Cultura, la Ciencia e Investigación, con el fin de contribuir a la satisfacción de las necesidades humanas, tanto de los estudiantes como de las comunidades. Específicamente la proyección social dentro de la corporación tiene cuatro pilares fundamentales: la práctica académica, la extensión, el emprendimiento y los egresado; esto está reglamentado mediante un acuerdo institucional numero $03-15$.

Dentro de los proyectos y actividades que ha desarrollado la corporación a lo largo de los años sea priorizado la intervención de comunidades vulnerables del departamento 
de sucre, para esto ha establecido acuerdos interinstitucionales y de cooperación con distintos organismos tanto públicos como privados, uno de sus proyectos bandera es el proyecto interinstitucional mundo mágico de MABA, del cual han participado diferentes facultades de la corporación. Este proyecto está enfocado hacia la atención integral en salud de niños en edad prescolar que pertenecen a hogares de escasos recursos; además este proyecto se ha ampliado hacia la capacitación de los padres de estos niños.

De otro lado la corporación ha realizado distintas actividades de atención integral desde convenios con instituciones como la unidad de víctimas, alcaldías municipales instituciones educativas, instituciones prestadoras de salud entre otras. La población beneficiaria de estos programas corresponde a distintos grupos de edad; como balance para el año 2015 la corporación beneficio a más de 1200 personas entre niños, niñas, adolecentes, adultos, adultos mayores e inclusive grupos de personas con diferentes condiciones especiales (discapacidad física y personas con necesidades especiales de aprendizajes), sin embargo su principal grupo de interés externo de atención son los niños y niñas en estado de vulnerabilidad socioeconómica. A continuación se presenta el diagrama 2 donde se muestra la participación que tienen los distintos grupos etarios en los programas de atención integral que desarrolla la Corporación.

Diagrama 2. Grupos de interés beneficiarios por los programas de extensión y proyección social de Corposucre, 2016

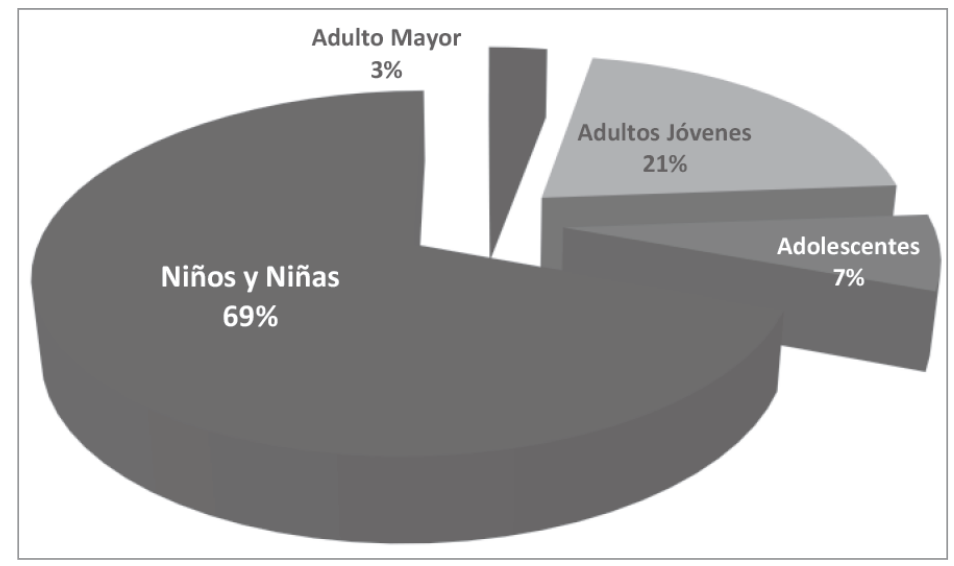

Fuente. Modulo de Proyección Social de Corposucre, 2016.

Por otra parte se muestran los resultados obtenido al avance en el diseño del programa de RSU para el emprendimiento sostenible en la corporación de los líderes de la comunidad encuestados (54), la mayoría son mujeres (el $68,5 \%$ ), con edades entre 31 y 40 años (el $35,2 \%)$, estos líderes en su mayoría ejercen la jefatura de hogar (el 61,1\%). El tamaño de los hogares en la urbanización está entre 3 y 4 integrantes (en la mitad de los casos); cabe destacar, que la urbanización Altos de la Sabana es un proyecto que está conformado, en su totalidad, por 137 edificios, cada uno con 16 apartamentos, los cuales, disponen de dos habitaciones, un baño y espacios comunes, un espacio de labores y una cocina, en un área total de 45,23 m2 por unidad residencial, lo cual, es un espacio reducido para mantener familias grandes; a pesar de esto, según los datos encontrados existe evidencia de 
familia ampliada, al menos, en el 5,6\% de los casos, llegando incluso a convivir en este tipo de viviendas, hasta ocho (8) personas, lo cual, por obvias razones crea condiciones de hacinamiento.

Estas familias tienen bajos niveles educativos, donde, la mayoría de los jefes de hogar (el 85,2\%), tienen como nivel máximo secundaria completa, mientras, que la mayoría de los conyugues (el 89,2\%) llegan también hasta este nivel como máximo; en el caso de los hijos, la situación no cambia mucho, donde, solo el 7,7\% de los jóvenes accede a educación superior. En la mayoría de estos hogares (el 70,4\%) existe una alta dependencia económica, donde, solo trabaja una sola persona, para mantener en promedio a tres (3) más, siendo, el conyugue y los hijos inactivos, donde, en el caso de estos últimos, la mayoría (el 94,4\%) aún se encuentra estudiando. De las personas que trabajan, la mayoría se dedica a labores informales, donde, predominan las actividades de albañilería y mototaxismo (ejercidas por hombres), labores domésticas (ejercidas por mujeres) y ventas en general (Ver Gráfico 1). Es de destacar, que estas personas ocupadas, en su mayoría (el 87,0\%), desde que trabajan siempre han ejercido este tipo de actividades, solo el 3,7\% tenía actividades netamente agrícolas.

Diagrama 3. Altos de la Sabana de Sincelejo. Tipo de Actividad Laboral. 2016.

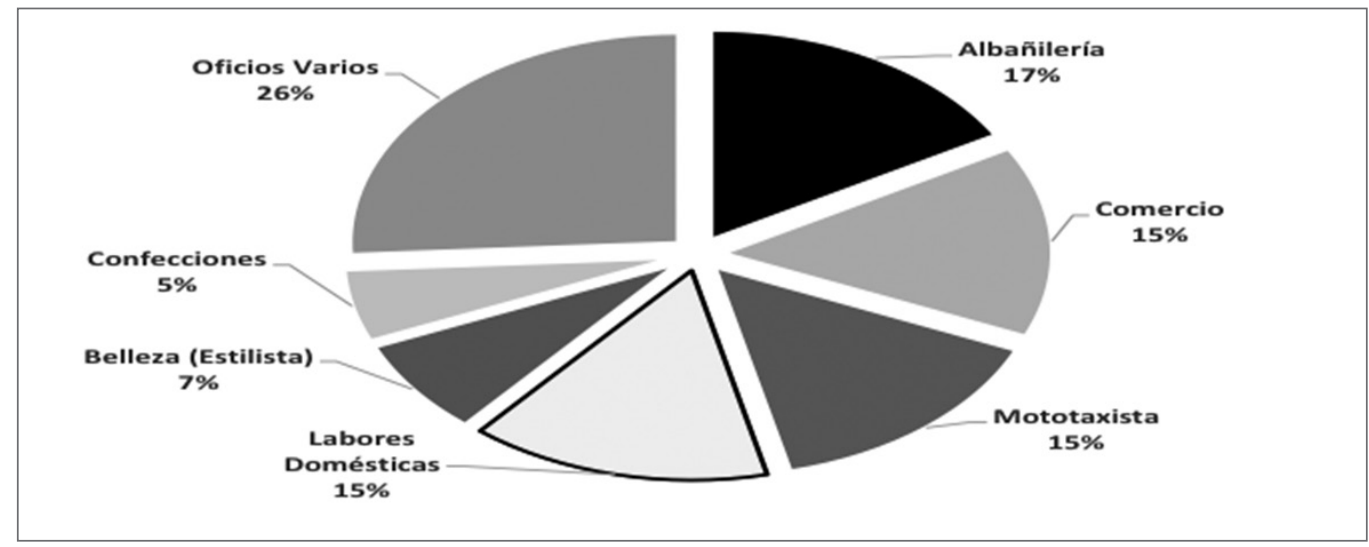

Fuente. Cálculos propios.

De las actividades que realizan los ocupados que fueron tomados para el estudio, solo el $22,2 \%$, se pueden considerar como actividades independientes, que pueden estar relacionadas con pequeños emprendimientos productivos; éstas son principalmente, salones de belleza, confecciones, comercio al por menor y labores de carpintería. Se debe destacar los ocupados, por lo general, ejercen actividades secundarias, donde, el tipo de oficio no varía mucho, con respecto al principal. En cuanto a formación para el trabajo, en el 53,5\% de los casos, la formación ha sido empírica, donde, otra persona, familiar o no, le enseñó el oficio; cabe destacar, que este tipo de conocimiento empírico puede ser utilizado para la apertura de emprendimientos productivos. En el 37,1\% de los casos, hay conocimientos que pueden ser oportunidades de negocio; la población tiene conocimiento en elaboración de productos alimenticios, cuidado y corte de cabello, confección de ropa, producción agropecuaria, mecánica automotriz y elaboración de artesanías; además, el 8,5\% de las personas 
encuestadas tienen conocimiento en sistemas e informática. Cabe destacar que dentro de los proyectos de vida de esta población, el 66,7\% de los ocupados afirmó que deseaba empezar un emprendimiento productivo, pero que no contaba con recursos financieros, ni técnicos para lograr este objetivo; en particular, el $22,2 \%$ desean recibir capacitación técnica o tecnológica que le permita potencializarse como emprendedor.

\section{Conclusiones}

Ne entiende que la responsabilidad social universitaria es una política fundamental que debe incorporarse en todas las universidades para la creación, y difusión del conocimiento científico en función de garantizar aporte de competencias cualidades y capacidades en las comunidades lo que traerá como respuesta el desarrollo sostenible, por lo cual la responsabilidad de las universidades trasciende de generar programas de altruismo o generosidad, a ejercer un proceso de gestión de conocimiento que la comprometa socialmente desde el mismo ejercicio de sus funciones primales.

Por consiguiente, se concibe la importancia en destacar que las universidades son organizaciones sociales que deben generar procesos de cambio para proporcionar mejoras en la colectividad, debido que es un asunto que debe pasar de la retórica a la practica con mayor frecuencia, cuando se desea justificar la necesidad de un programa académico este debe responder e impactar en las necesidades de la región donde se espera desarrollar procesos de cambio; por lo tanto se diseña un programa de responsabilidad social universitaria para la generación de emprendimiento sostenible que responde a las necesidades locales y apegado a los principios universitarios considerando un elemento fundamental que es la vulnerabilidad propia de la comunidad.
En tal sentido el compromiso de la corporacion universitaria Antonio José de Sucre es operar desde la misma comunidad para generar el impulso de la creación de iniciativas de negocios que satisfagan las necesidades de la sociedad, incrementando la calidad de vida de las personas, desarrollando sinergia entre la universidad, la comunidad, los entes gubernamentales y el sector empresarial. Es así que para el diseño de un programa de responsabilidad social en las comunidades vulnerables, se requiere de la intervención de distintas instituciones, tanto públicas como privadas, para que más que una ayuda asistencialista, se generen en ellas capacidades, como forma de autonomía y dignificación de sus condiciones, donde el emprendimiento sostenible es una estrategia de empoderamiento para que las comunidades generen sus propios beneficios económicos elevando así su calidad de vida.

De tal manera se logró identificar que grupo de líderes Altos de la Sabana, es una población joven, relativamente feminizada, en cuyos hogares predomina la familia nuclear, la cual, tiene bajos niveles educativos, poco acceso a educación superior; donde, existe alta dependencia económica y donde, sus ocupados ejercen actividades laborales informales y de subsistencia, a pesar que están en una edad altamente productiva, pero no tienen los conocimientos así como niveles de formación adecuados, por lo que quedan expulsados del mercado laboral. 
En este caso específico de Corposucre se puede evidenciar una amplia gama de actividades proyectos y líneas estratégicas bajo acuerdos normativos que se configuran como parte de la extensión y proyección social que ha asumido la corporación, sin embargo el concepto de responsabilidad social universitaria no se alcanza a dimensionar en toda su extensión, debido que aún no existe una claridad acerca del mismo quedando de esta forma las acciones implementadas por la corporación dentro de grupos vulnerables como actividades desconectadas y discrecionales e incluso desde un espíritu altruista, y no como una política transversal de acción por parte de la corporación hacia los grupos de interés.

Finalmente es relevante destacar que los habitantes de la comunidad altos de la sabana tienen ímpetu y características para iniciar emprendimiento social sostenible pero es importante que los entes gubernamentales se acerquen más a ellos, ya que manifiestan tener poca participación en los espacios del gobierno local, regional y nacional, por tal motivo se sienten aislados y no sienten la asistencia y apoyo que tanto necesitan para dar respuesta a sus necesidades. 


\section{Referencias bibliográficas}

Ander, E. (2008). Técnicas de Investigación Social. Editorial Humanitas Buenos Aires XXII Edición

Bernal, H. \& Rivera, B. (Dir.), (2011). Responsabilidad social universitaria: Aportes para el análisis de un concepto. Responsabilidad Social Universitaria. El Pensamiento Universitario $N^{\circ} 21$. Documentos. Asociación Colombiana de Universidades - ASCUN. ISSN. 0124-3543. Bogotá D.C., marzo de 2011. pp. 7 - 17. (105).

Blanco, A. \& Chacón, F. (1985). La evaluación de la calidad de vida. En J. F. Morales, J. F.; Blanco, A.; Huici, C. y Fernández, J. M. (Eds.), Psicología Social Aplicada. Madrid: Morata.

Bueno, E. (2013). Pobreza multidimensional y vulnerabilidad social. Universidad de Zacatecas. Observatorio del Desarrollo, volumen 1, número 4. México, abril de 2013. pp. 38-45.

Busso, G. (2001). Vulnerabilidad social: nociones e implicancias de políticas para Latinoamérica a inicios del Siglo XXI. Comisión Económica para América Latina y el Caribe - CEPAL, Centro Latinoamericano y Caribeño de Democracia - CELADE. Seminario Internacional, las diferentes expresiones de la vulnerabilidad social en América Latina y el Caribe. Santiago de Chile, junio de 2001.

Corposucre, (2015). Acuerdo No 03-15, 11 de marzo de 2015. Por el cual se reglamenta la organización y desarrollo de la Proyección Social de la Corporación Universitaria Antonio José de Sucre. (27).
Chirinos, Y; (2014). Capital Humano Factor potencial para el Emprendimiento Sostenible. Revista Impacto Científico Núcleo LUZ - COL Volumen 9, No 2, Diciembre 2014. ISSN 1856- 5049. pp. 206 $-306$.

Chirinos, Y., \& RamosL; (2014). Emprendimiento Sostenible como política de Estado. Libro electrónico Tendencias y Perspectivas de Políticas Públicas en Ciencia Tecnología e Innovación Volumen I, No 1 año 2014. ISBN 968-980-402-160-2. pp. 305 - 324.

Cohen, E \& Franco, R. (2005). Gestión Social: Como Lograr Eficiencia e Impacto en las Políticas Sociales. Editorial siglo XXI S.A. En coedición con Naciones Unidas. México.

Cordero, M., (2011). Desarrollo Inclusivo Basado en Comunidad: una estrategia para la inclusión social de las personas con discapacidad y el desarrollo de las comunidades desde el enfoque de derechos humanos. Costa Rica, enero de 2011. p. 88.

Correa, C., (2011). El emprendimiento y empresarismo en las Mipymes, frustración o éxito. I Foro Intersectorial Unicafam: la investigación y la competitividad Mipyme. Fundación Universitaria Cafam. (14). Recuperado de

h t t p : / / ww w. unicafam. ed u. co/ EventoAcademico_1/flash/Articulos/a8.pdf

Espinosa, M., Calzoncit, J., Valdez, M. \& Castro, G., (2012). Los trabajadores sociales ¿un grupo vulnerable?, en: La problemática de los grupos vulnerables: visiones de la realidad. Octubre de 2012. pp. 10-33. 
Fuller, N. (2006). Management development in small firms, en international journal of Management Review. N. 8-3. pp. $175-190$.

Hernández, R., Fernández, C., \& Baptista, P. (2010). Metodología de la Investigación. México D.F.: Mcgraw-HILL / Interamericana Editores, S.A. de C.V

Ishikawa, A. \& Strandberg, L., (2009). Negocios Inclusivos: Creando valor para las empresas y para la población de bajos ingresos. Cuadernos de la Cátedra "La Caixa" de Responsabilidad Social de la Empresa y Gobierno Corporativo. N ${ }^{\circ}$ 5, noviembre de 2009. IESE Business School - Universidad de Navarra. pp. 32.

Kantis, H., Federico, J. \& Menéndez, C., (2012). Políticas de fomento al emprendimiento dinámico en América Latina: tendencias y desafíos. CAF, Documento de Trabajo, N²012/09, Caracas, Agosto de 2012. (53).

López, J. (2012). Modelos actitudinales y Emprendimiento Sostenible. Cuaderno Interdisciplinar de Desarrollo Sostenible. Revista Cuadernos interdisciplinar de desarrollo sostenible. Vol. I, No. 8: Pp. 111131 http://repositorio.ual.es:8080/jspui/ bitstream/10835/1402/7/Act_Emp_Sost. pdf

Marrero, A., (2007). La sociedad del conocimiento: una revisión teórica de un modelo de desarrollo posible para América Latina. Arxius, Número 17, Desembre 07, Universidad de la República, Uruguay. pp. 63-73. (11).

Misas, G., (2004). La educación superior en Colombia. Análisis y estrategias para su desarrollo. Universidad Nacional de Colombia, ISBN: 958-701-356-5. p. 298.
Naidorf, J., Giordana, P. \& Horn, M., (2007). La pertinencia social de la universidad como categoría equívoca. Nómadas (Col), núm. 27, octubre de 2007. ISSN (Versión impresa): 0121-7550. Universidad Central, Bogotá. pp. 22-33.

Rama, C., (2007). Los nuevos estudiantes en circuitos diferenciados de educación. En Educación superior en América Latina y el Caribe: Sus estudiantes hoy. Colección Idea Latinoamericana, Unión de Universidades de América Latina y el Caribe, México, 2007, pp. $13-42$.

Rodríguez, J, (2012). La Responsabilidad Social es inherente a la naturaleza y misión de la Universidad; en: Domínguez, Julio \& Rama, Claudio, (Edit.). La Responsabilidad Social Universitaria en la Educación a Distancia. Universidad Católica Los Ángeles de Chimbote - Universidad de la Empresa Soriano. ISBN: 978-612-45269-8-5, D. Leg. No 2012-05672. (258).

Sánchez, A., (2008). La Formación de Emprendedores Sociales: Caso Tecnológico de Monterrey. Ide@s CONCYTEG, Año 3; Núm. 33, marzo de 2008. Recuperado de https://www.researchgate.net/ publication/267854317_La_Formacion_ de_Emprendedores_Sociales_Caso_ Tecnologico_de_Monterrey

Suárez, G., (2007). Presupuestos y realidades para la investigación en la universidad colombiana. Pontificia Universidad Javeriana. Studiositas, 2(3). ISSN: 1909-0366. Bogotá, septiembre de 2007. pp. 54-66. (13).

Sparano, H. (2014). Emprendimiento en América Latina y su impacto en la gestión de proyectos. Revista Dimensión Empresarial, vol. 12, núm. 2. pp. 95-106. 
Tünnermann, C. \& De Souza, M., (2003). Desafíos de la universidad en la sociedad del conocimiento, Cinco años después de la conferencia mundial sobre educación superior. UNESCO Forum Occasional Paper Series, Paper $n^{\circ} 4 / \mathrm{S}$. París, diciembre de 2003. (31).

Unesco, (2008). Educación superior y sociedad. El movimiento de la responsabilidad social de la universidad: una comprensión novedosa de la misión universitaria. Nueva Época. Año 13, Número 2. SSN 07981228. Caracas, septiembre de 2008. (206).

Uninorte - Universidad del Norte, et al., (2016). GEM Colombia 2014. Global Entrepreneurship Monitor Colombia.
ISBN: 978-958-741-661-9. Xpress Estudio Gráfico, Bogotá - Colombia. (120).

Urzúa, A. \& Caqueo-Urízar, A. (2012). Calidad de vida: una revisión teórica del concepto. Revista Terapia Psicológica, Vol. 30, n¹, Santiago de Chile, abril de 2012. pp. 61-71.

Vallaeys, F. (2010). Breve marco teórico de responsabilidad social universitaria. Breve marco teórico de responsabilidad social universitaria. en CD: Responsabilidad social universitaria, Red Universitaria de Ética y Desarrollo Social (RED), Iniciativa Interamericana de Capital Social, Ética y Desarrollo delBID.(10). Recuperado dehttp:// rsuniversitaria.org/web/images/stories/ 
